\title{
Pentoxifylline exerts anti-inflammatory effects on cerebral ischemia reperfusion-induced injury in a rat model via the p38 mitogen-activated protein kinase signaling pathway
}

\author{
JIANQING DONG, XINMEI YUAN and WEIWEI XIE
}

Department of Blood Transfusion, Zhangqiu People's Hospital, Jinan, Shandong 250200, P.R. China

Received October 7, 2016; Accepted July 27, 2017

DOI: $10.3892 / \mathrm{mmr} .2017 .7953$

\begin{abstract}
Pentoxifylline exhibits complex functions with extensive pharmacological effects and is used therapeutically due to its therapeutic effects and rapid metabolism in the body, with no cumulative effects and few side effects. The present study investigated the effects of pentoxifylline on cerebral ischemia reperfusion-induced injury (IRI) through suppression of inflammation in rats. Hematoxylin and eosin staining was performed to evaluate the number of neurocytes, and ELISAs were applied to measure tumor necrosis factor- $\alpha$, interleukin-6, malondialdehyde and superoxide dismutase activities. Treatment with pentoxifylline significantly recovered the cerebral ischemia reperfusion-induced neurological deficit score and cerebral infarct volume in rats. In addition, pentoxifylline treatment significantly reversed the cerebral ischemia reperfusion-induced interleukin-6, tumor necrosis factor- $\alpha$, malondialdehyde and superoxide dismutase levels in vivo. Furthermore, pentoxifylline significantly inhibited cyclooxygenase-2 and inducible nitric oxide synthase mRNA and protein expression in cerebral IRI mice. Treatment with pentoxifylline also significantly suppressed the expression of cleaved caspase- 3 and p38 mitogen-activated protein kinase (MAPK) protein in cerebral IRI mice. These results indicate that the protective effects of pentoxifylline on cerebral IRI may occur via the p38 MAPK signaling pathway.
\end{abstract}

\section{Introduction}

Cerebrovascular disease poses a substantial threat to human health. Annually, 1.5 million new patients are diagnosed in China and the most common type is ischemic stroke, namely cerebral ischemia (1). In recent years, incidence trends have shifted towards younger individuals, and the majority of

Correspondence to: Dr Jianqing Dong, Department of Blood Transfusion, Zhangqiu People's Hospital, 1920 Huiquan Road, Zhangqiu, Jinan, Shandong 250200, P.R. China

E-mail: sijueqinju86569813@126.com

Key words: pentoxifylline, cerebral ischemia reperfusion-induced injury, p38 mitogen-activated protein kinase patients experience nerve function defect following the attack, which greatly affects the quality of life of patients and is associated with economic and mental burdens to society and the family and friends of patients (2). However, at present, no effective treatment exists.

Cerebral ischemia reperfusion-induced injury (IRI) is a highly complex pathological process that involves a series of cellular and molecular events (3). It is divided into the following three stages: Acute stage, which primarily manifests as metabolic disorders and excitatory toxicity; sub-acute stage, where the major pathological changes are inflammation and apoptosis; and chronic stage, which primarily consists of repair and regeneration (4). The sub-acute and chronic stages are also collectively termed the 'late stage', as the boundary between the two stages is difficult to determine (5).

A previous study demonstrated that inflammation has an important role in cerebral IRI (6). The early accumulation of neutrophil granulocytes at ischemic regions has been verified by histopathology and biochemical methods (7). The most frequently used reliability index to evaluate the degree of neutrophil granulocyte infiltration in tissue is myeloperoxidase (8), which is an enzyme that catalyzes peroxide reduction and is an essential constituent of the oxygen-dependent sterilization system of neutrophil granulocytes (9).

Pentoxifylline is a type of alkaloid that is formed by introducing a hexanone group to theobromine extracted from cocoa beans (10). It is a derivative of methylxanthine and non-selective phosphodiesterase inhibitors (11). Pentoxifylline has been reported to increase the deformability of red blood cells, improve the hemorheology of leukocytes, inhibit the adhesion and activation of neutrophil granulocytes, expand capillaries, reduce blood viscosity, increase the oxygen partial pressure of tissue and eliminate free radicals $(12,13)$. Therefore, the present study was performed to investigate the protective effects of pentoxifylline on cerebral IRI and the potential underlying mechanisms.

\section{Materials and methods}

Animals and treatments. Male Sprague-Dawley rats weighing 260-300 g ( $\mathrm{n}=36 ; 1.5$ years old) were obtained from the Experimental Animal Centre of Zhangqiu People's Hospital (Jinan, China) and were allowed free access to laboratory chow and tap water in day-night quarters at $25^{\circ} \mathrm{C}$ with 
50-60\% humidity and a 12-h light/dark cycle. The experiment was approved by the Committee on Animal Experiments of Zhangqiu People's Hospital (14). All rats were randomly divided into the following three experimental groups ( $n=12$ per group): Sham, sham-operated rats pretreated intraperitoneally with normal saline for 3 days; cerebral IRI model, cerebral IRI model rats pretreated intraperitoneally with normal saline for 3 days; and pentoxifylline treatment group, cerebral IRI model rats pretreated daily with $46.7 \mathrm{mg} / \mathrm{kg}$ pentoxifylline (Sigma-Aldrich; Merck KGaA, Darmstadt, Germany) intraperitoneally for 3 days. The chemical structure of pentoxifylline (3,7-dimethyl-1-(5-oxo hexyl)-3,7-dihydro-1H-purine-2,6-dione) is presented in Fig. 1.

Cerebral IRI model. Intraluminal filamentous occlusion of the middle cerebral artery (MCA) was performed to induce the cerebral IRI model for $1 \mathrm{~h}$. Reperfusion of the MCA was initiated by removing the MCA-occlusive filament after the $1 \mathrm{~h}$ of occlusion and then reperfusion was performed for $1 \mathrm{~h}$. The mean arterial blood pressure, arterial blood gases and $\mathrm{pH}$ were monitored following cannulation of the right femoral artery. A laser Doppler flowmeter (Periflux System 5000; Perimed AB, Järfälla, Sweden) was used to monitor regional cerebral blood flow. Sham-operated rats were anesthetized (35 mg/kg pentobarbital sodium) and surgically opened up without cerebral IRI induction. Brain samples were fixed using $4 \%$ paraformaldehyde for $24 \mathrm{~h}$ at room temperature and processed by routine histological methods, embedded in paraffin blocks and sectioned coronally into sequential $5-\mu \mathrm{m}$ sections. Then, section samples were stained using haematoxylin and eosin for $20 \mathrm{~min}$ at room temperature. Subsequently, neurological deficit scores were analyzed $(0$, normal; 1 , moderate; 2 , considerable; 3 , severe) following treatment with pentoxifylline for 3 days using a fluorescence microscope (OLYMPUS BX51; Olympus Corporation, Tokyo, Japan).

Evaluation of cerebral infarct volume. Rats were sacrificed by decapitation and cerebral infarct tissues were rapidly acquired. Cerebral infarct tissues were frozen at $-80^{\circ} \mathrm{C}$ for storage. Subsequently, cerebral infarct tissues were fixed with $4 \%$ paraformaldehyde in PBS for $24 \mathrm{~h}$ at room temperature. Then, brains tissues were sliced into $5-\mu \mathrm{m}$ uniform coronal sections, which were stained with $2 \%$ 2,3,5-triphenyltetrazolium chloride solution for $1 \mathrm{~h}$ at $37^{\circ} \mathrm{C}$. Cerebral infarct volume was using a fluorescence microscope (OLYMPUS BX51) and measured using OlyVIA software version 2.6 (both Olympus Corporation).

Histology. Rats were sacrificed by decapitation and cerebral infarct tissues were rapidly acquired. Cerebral infarct tissues were fixed in $3 \%$ paraformaldehyde in PBS for $4 \mathrm{~h}$ on ice and then transferred to $50 \%$ ethanol for $2 \mathrm{~h}$. Tissues samples were cut into histological sections $(4-\mu \mathrm{m})$ and were stained with hematoxylin and eosin (H\&E) for $15 \mathrm{~min}$ at room temperature using light microscope (magnification, x20; Metallurgical Microscope; Shanghai Optical Instrument Import \& Export Co., Ltd., Shanghai, China) to analyze the number of neurocytes.

Evaluation of tumor necrosis factor (TNF)- $\alpha$, interleukin (IL)-6, malondialdehyde (MDA) and superoxide dismutase (SOD) activities. Rats were sacrificed by decapitation and

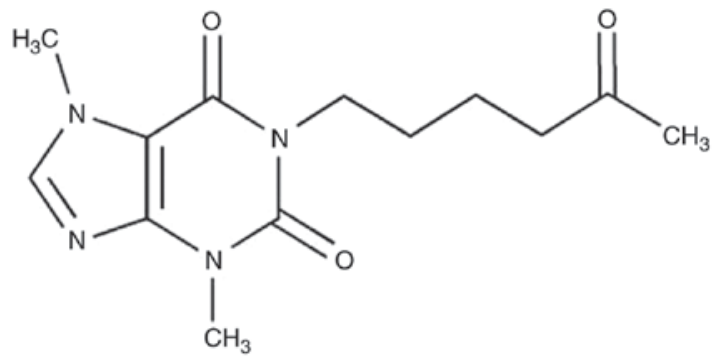

Figure 1. The chemical structure of pentoxifylline.

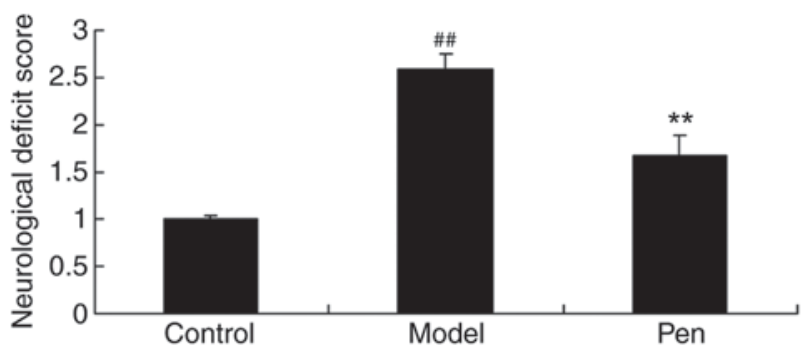

Figure 2. Protective effects of pentoxifylline on neurological deficit score. ${ }^{\# \#} \mathrm{P}<0.01$ vs. control group; ${ }^{* *} \mathrm{P}<0.01$ vs. model group. Control, sham-operated group; model, cerebral ischemia reperfusion injury model group; pen, pentoxifylline-treated group.

blood samples $(0.5 \mathrm{ml})$ were collected from the femoral vein. Serum was isolated from the blood following centrifugation at $1,000 \mathrm{x} \mathrm{g}$ for $20 \mathrm{~min}$ at $4^{\circ} \mathrm{C}$. ELISA analyses were performed using ELISA kits for TNF- $\alpha$ (cat. no. KRC3012; Biosource; Thermo Fisher Scientific, Inc., Waltham, MA, USA), IL-6 (cat. no. BMS625TEN; Biosource; Thermo Fisher Scientific, Inc.), MDA (cat. no. A003-1; Nanjing Jiancheng Bioengineering Institute, Nanjing, China) and SOD (cat. no. A001-1-1; Nanjing Jiancheng Bioengineering Institute) to measure their activities in serum samples, according to the manufacturer's protocol.

Measurement of cyclooxygenase (COX)-2 and inducible nitric oxide synthase (iNOS) mRNA expression. Total RNA was prepared from cerebral infarct tissues using TRIzol reagent (Invitrogen; Thermo Fisher Scientific, Inc.) according to the manufacturer's protocol. RNA samples $(1 \mathrm{mg})$ were used for cDNA synthesis, which was performed using the RevertAid First Strand cDNA Synthesis kit (Fermentas; Thermo Fisher Scientific, Inc.) according to the manufacturer's instructions. The temperature protocol was as follows: $37^{\circ} \mathrm{C}$ for $1 \mathrm{~h}$ and $42^{\circ} \mathrm{C}$ for $10 \mathrm{~min}$. Quantitative (q)PCR was performed using SYBR Green PCR Master Mix (Applied Biosystems; Thermo Fisher Scientific, Inc.) by the ABI 7500 system (Applied Biosystems; Thermo Fisher Scientific, Inc.) by to measure the mRNA expression of COX-2 and iNOS $(n=3)$. The following primers were used: COX-2 sense, 5'-GTGGGA TGACGAGCGACTGT-3' and antisense 5'-TTTCAGGGA GAAGCGTTTGC-3'; iNOS sense, 5'-GCATCCCAAGTA CGAGTGGT-3' and antisense 5'-GAAGGCGTAGCTGAA CAAGG-3'; and GAPDH sense, 5'-CCATCACTGCCACTC AGAAGA-3' and antisense 5'-CATGAGGTCCACCACCCT GT-3'. The PCR conditions were $94^{\circ} \mathrm{C}$ for $5 \mathrm{~min}, 35$ cycles of $94^{\circ} \mathrm{C}$ for $30 \mathrm{sec}, 55^{\circ} \mathrm{C}$ for $30 \mathrm{sec}$ and $72^{\circ} \mathrm{C}$ for $1 \mathrm{~min}$, and then 

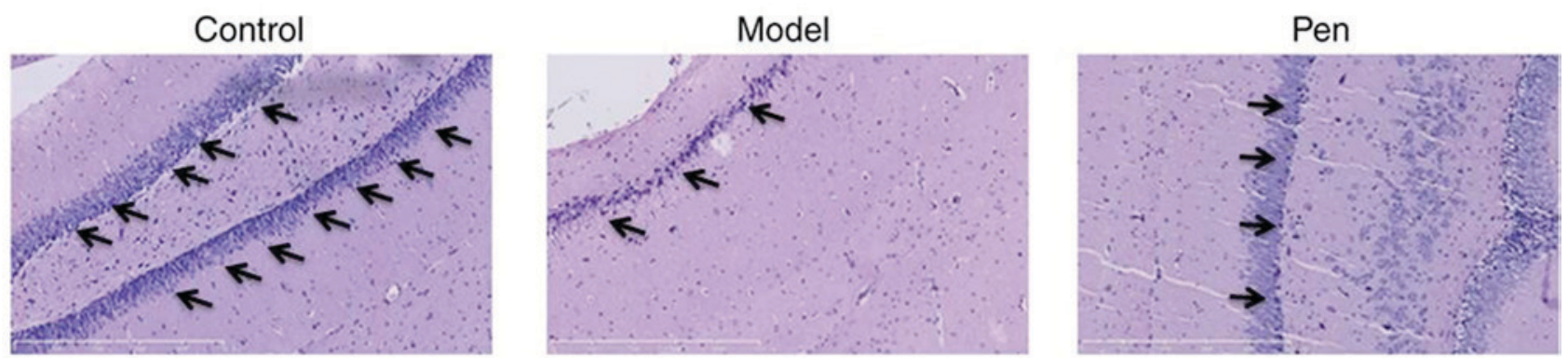

Figure 3. Protective effects of pentoxifylline on neurocytes. Tissues were stained with hematoxylin and eosin (magnification, x20). Control, sham-operated group; model, cerebral ischemia reperfusion injury model group; pen, pentoxifylline-treated group.

$4^{\circ} \mathrm{C}$ for $10 \mathrm{~min}$. miRNA expression were measured using the $2^{-\Delta \Delta C t}$ method (15). Experiments were repeated three times.

Western blotting. Rats were sacrificed by decapitation and cerebral infarct tissues were rapidly acquired. Cerebral infarct tissues were frozen at $-80^{\circ} \mathrm{C}$. Subsequently, brain tissues were ground into a powder with liquid nitrogen and lysed in radioimmunoprecipitation assay lysis buffer (Beyotime Institute of Biotechnology, Haimen, China) for 30 min at $4^{\circ} \mathrm{C}$, which was followed by centrifugation at $12,000 \mathrm{xg}$ for $5 \mathrm{~min}$ at $4^{\circ} \mathrm{C}$. The supernatant was collected and the protein concentration was determined using a BCA Protein assay kit (Sangon Biotech Co., Ltd., Shanghai, China). Proteins $(50 \mu \mathrm{g})$ were separated on $12 \%$ SDS polyacrylamide gels (Sangon Biotech Co., Ltd.) and electrophoretically transferred to polyvinylidene difluoride membranes (EMD Millipore, Billerica, MA, USA), which were then blocked using 5\% non-fat milk in TBST (TBS containing $0.1 \%$ Tween-20) for $1 \mathrm{~h}$ at $37^{\circ} \mathrm{C}$. Membranes were incubated overnight at $4^{\circ} \mathrm{C}$ with anti-COX-2 (cat. no. sc-7951; 1:2,000; Santa Cruz Biotechnology), anti-iNOS (cat. no. sc-649; 1:2,000; Santa Cruz Biotechnology), anti-cleaved caspase-3 (cat. no. 9662; 1:2,000; Santa Cruz Biotechnology), anti-matrix metallopeptidase (MMP)-9 (cat. no. sc-10737; 1:2,000; Santa Cruz Biotechnology), anti-phosphorylated (p)-p38 mitogen-activated protein kinase (cat. no. 4511; 1:3,000; Cell Signaling Technology, Inc.) and anti- GAPDH (cat. no. sc-25778; 1:500; Santa Cruz Biotechnology). Membranes were incubated with goat anti-rabbit horseradish peroxidase-conjugated IgG (cat. no. sc-2004; 1:5,000; Santa Cruz Biotechnology) for $1 \mathrm{~h}$ at $37^{\circ} \mathrm{C}$. Protein expression was observed with the BeyoECL Plus chemiluminescence reagent (Beyotime Institute of Biotechnology) and analyzed using Image_Lab_3.0 (Bio-Rad Laboratories, Inc., Hercules, CA, USA).

Statistical analysis. Data are presented as the mean + standard error of the mean. Statistical analysis was performed by one-way analysis of variance and Dunnett's post-hoc test, which was performed using SPSS 17.0 software (SPSS, Inc., Chicago, IL, USA). $\mathrm{P}<0.05$ was considered to indicate a statistically significant difference.

\section{Results}

Protective effects of pentoxifylline on neurological deficit score. To determine the protective effects of pentoxifylline

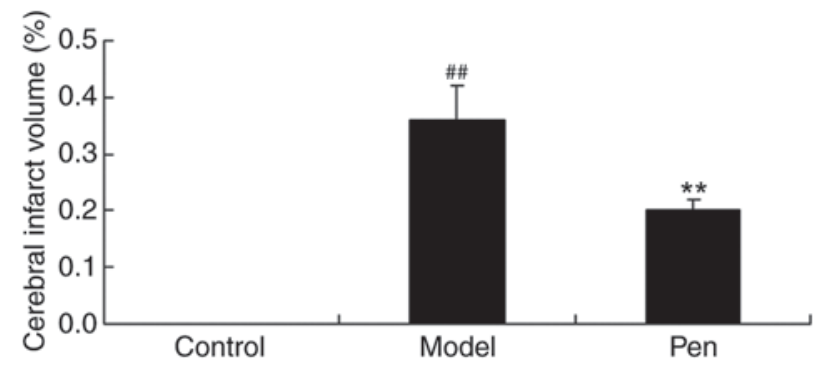

Figure 4. Protective effects of pentoxifylline on cerebral infarct volume. ${ }^{\# \#} \mathrm{P}<0.01$ vs. control group; ${ }^{* *} \mathrm{P}<0.01$ vs. model group. Control, sham-operated group; model, cerebral ischemia reperfusion injury model group; pen, pentoxifylline-treated group.

on neurological deficit score, neurological deficit scores were evaluated. As presented in Fig. 2, there was a significant increase in the neurological deficit score of the cerebral IRI model group, compared with the sham-operated rats (control) group. However, pretreatment with pentoxifylline significantly reduced the neurological deficit score of cerebral IRI rats (Fig. 2).

Protective effects of pentoxifylline on neurocytes. To further determine the protective effects of pentoxifylline on neurocytes, neurocytes in brain sections were stained using H\&E. The number of neurocytes in the sham group was higher compared with the cerebral IRI model group (Fig. 3). However, pentoxifylline treatment increased neurocytes compared with the cerebral IRI model group (Fig. 3).

Protective effects of pentoxifylline on cerebral infarct volume. The present study also determined the protective effects of pentoxifylline on cerebral infarct volume. In the sham group, cerebral infarct volume was lower compared with the cerebral IRI model group (Fig. 4). However, treatment with pentoxifylline significantly reduced the cerebral infarct volume in rats with cerebral IRI (Fig. 4).

Effects of pentoxifylline on IL- 6 and TNF- $\alpha$ levels in cerebral IRI rats. The present study also determined the effects of pentoxifylline on IL-6 and TNF- $\alpha$ levels in the serum of cerebral IRI rats using ELISA kits. As demonstrated in Fig. 5, a significant increase in the levels of TNF- $\alpha$ and IL-6 was observed in cerebral IRI rats compared with the sham-operated group. Pretreatment with pentoxifylline 
A

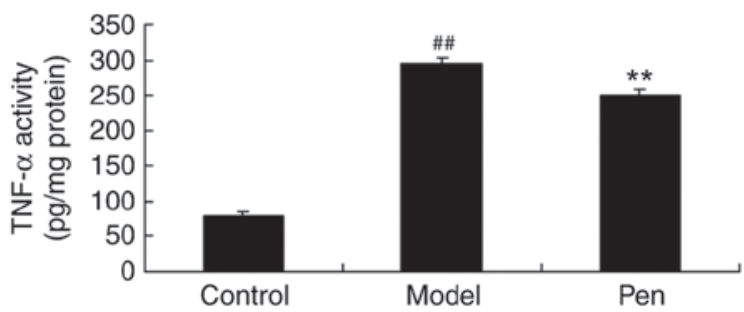

B

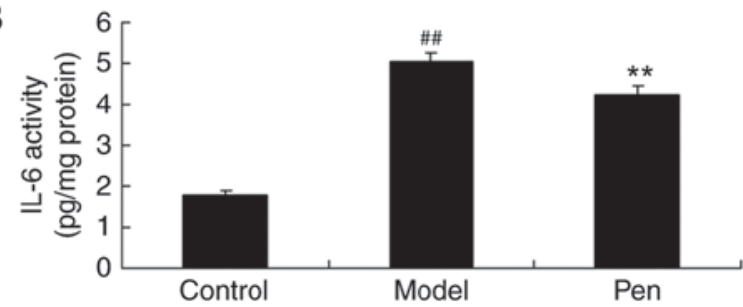

Figure 5. Pentoxifylline treatment affects IL-6 and TNF- $\alpha$ serum levels in cerebral IRI rats. Effects of pentoxifylline on (A) TNF- $\alpha$ and (B) IL-6 levels in cerebral IRI rats. ${ }^{\# \#} \mathrm{P}<0.01$ vs. control group; ${ }^{* *} \mathrm{P}<0.01$ vs. model group. TNF, tumor necrosis factor; IL, interleukin; IRI, ischemia reperfusion injury; control, sham-operated group; model, cerebral ischemia reperfusion injury model group; pen, pentoxifylline-treated group.

A

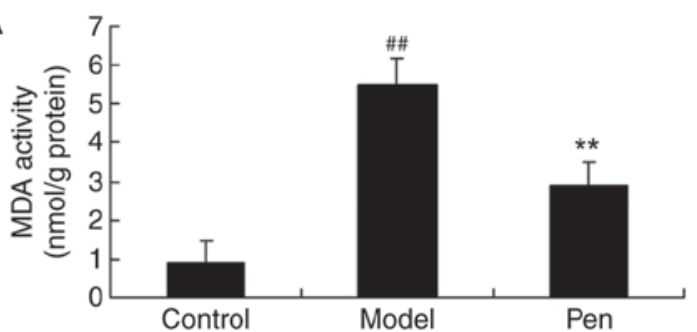

B

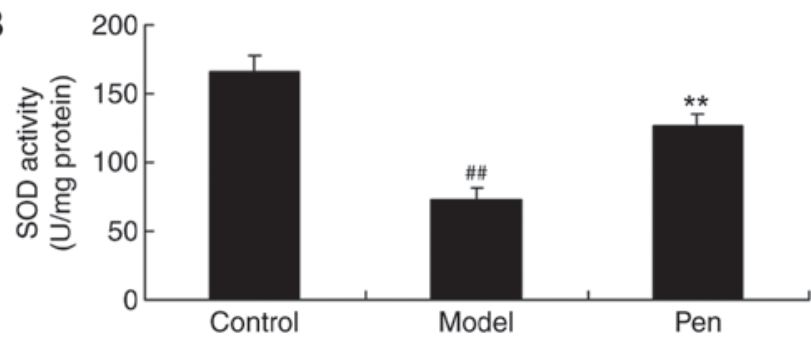

Figure 6. Pentoxifylline treatment affects MDA and SOD levels in cerebral IRI rats. Effects of pentoxifylline on (A) MDA and (B) SOD levels in cerebral IRI rats. ${ }^{\# \#} \mathrm{P}<0.01$ vs. control group; ${ }^{* *} \mathrm{P}<0.01$ vs. model group. MDA, malondialdehyde; SOD, superoxide dismutase; IRI, ischemia reperfusion injury; control, sham-operated group; model, cerebral ischemia reperfusion injury model group; pen, pentoxifylline-treated group.

significantly suppressed the levels of IL- 6 and TNF- $\alpha$ in the serum of cerebral IRI rats (Fig. 5).

Effects of pentoxifylline on MDA and SOD levels in cerebral IRI rats. Furthermore, the effects of pentoxifylline on MDA and SOD levels in the serum of cerebral IRI rats were investigated by ELISA. Compared with the sham-operated rats, there was a significant increase in MDA and reduction in SOD levels in the serum of cerebral IRI model rats (Fig. 6). However, the MDA and SOD levels were significantly reversed by treatment with pentoxifylline in cerebral IRI rats (Fig. 6).

Effects of pentoxifylline on COX-2 and iNOS mRNA and protein expression in cerebral IRI rats. Reverse transcription-qPCR (RT-qPCR) analysis and western blotting was performed to determine the changes in COX-2 and iNOS mRNA and protein expression, respectively. The sham-operated group exhibited a significant decrease in COX-2 and iNOS mRNA and protein expression, compared with cerebral IRI model rats (Fig. 7). However, treatment with pentoxifylline significantly suppressed the COX-2 and iNOS mRNA and protein expression in cerebral IRI rats (Fig. 7).

Effects of pentoxifylline on cleaved caspase-3, MMP-9 and 38 protein expression in cerebral IRI rats. In order to determine the effects of pentoxifylline on cleaved caspase-3, MMP-9 and p-p38 MAPK protein expression in cerebral RI rats, western blotting was performed. The protein expression of cleaved caspase- 3 and p-p38 MAPK was significantly increased in cerebral IRI rats, while the expression of MMP-9 was significantly reduced, compared with sham control rats (Fig. 8). However, the protein expression of cleaved caspase-3,
MMP-9 and p38 was significantly reversed with pentoxifylline administration in cerebral IRI rats (Fig. 8).

\section{Discussion}

Ischemic cerebral vascular disease is a common neurological disease, and its high disability and death rates negatively affect families and society (16). At present, the principle of treatment is to restore the blood supply to the ischemic area, however, the reperfusion injury that follows this may exacerbate the brain dysfunction and cause further damage to the tissue, which is termed IRI injury (17). IRI often occurs in the recanalization of cerebral vascular embolization, heart failure correction, shock correction, IRI microcirculation recanalization and cardiopulmonary-cerebral resuscitation (18). With the increased understanding of the pathophysiological mechanism of cerebral IRI, the inhibition of reperfusion injury has become an important part of the treatment of ischemic cerebrovascular disease (19), and the effectiveness and safety of these treatments is the focus in the clinic. In the present study, pretreatment with pentoxifylline significantly reduced the neurological deficit score and cerebral infarct volume in rats with cerebral IRI. Taken together, these results indicate that pentoxifylline may be a potential candidate drug for cerebral IRI.

COX is a type of rate-limiting enzyme that catalyzes the synthesis of prostaglandin and thromboxane from arachidonic acid (20). There are two isomers of COX; COX-1 exists in the majority of tissues as a structural type and catalyzes the production of prostaglandins required to maintain the normal physiological function $(21,22)$, and COX-2, which is abnormally expressed following cerebral ischemia, is a key enzyme in the production of free radicals and inflammatory mediators, and is involved in the development of ischemic brain 


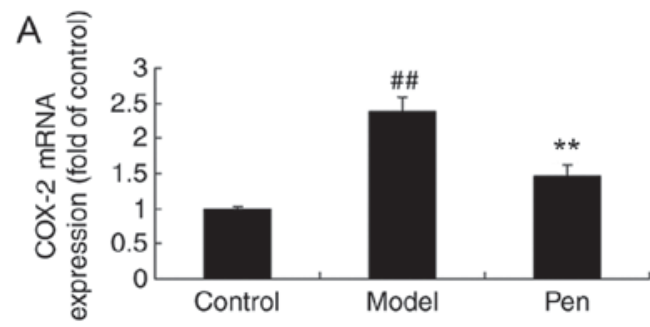

B
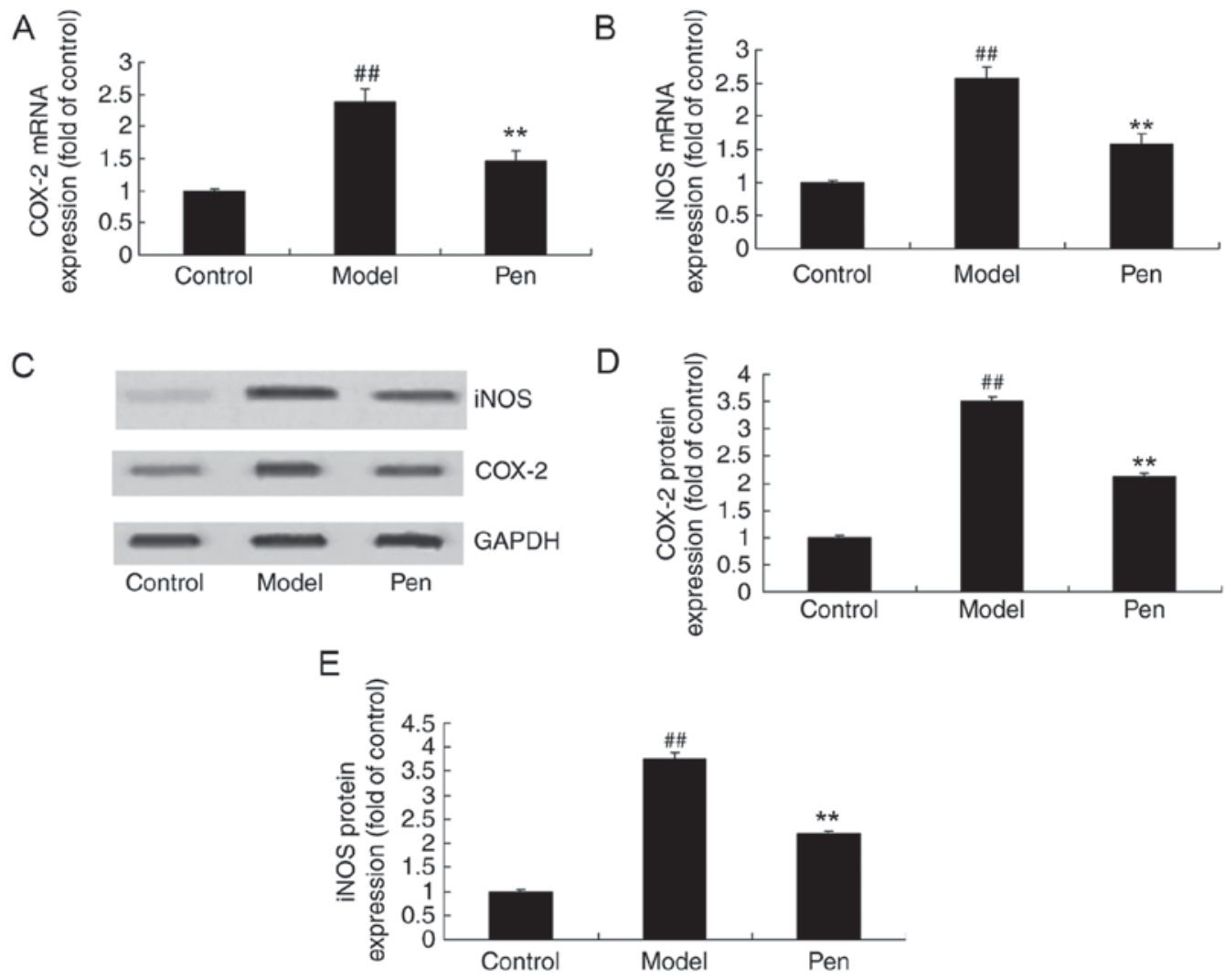

Figure 7. Pentoxifylline treatment affects COX-2 and iNOS mRNA and protein expression in cerebral IRI rats. Effects of pentoxifylline on the mRNA expression of (A) COX-2 and (B) iNOS. (C) Representative western blot for COX-2 and iNOS protein expression. Densitometric and statistical analysis of (D) COX-2 and (E) iNOS protein expression in cerebral IRI rats. ${ }^{\# \#} \mathrm{P}<0.01$ vs. control group; ${ }^{* *} \mathrm{P}<0.01$ vs. model group. COX, cyclooxygenase; iNOS, inducible nitric oxide synthase; IRI, ischemia reperfusion injury; control, sham-operated group; model, cerebral ischemia reperfusion injury model group; pen, pentoxifylline-treated group.

A

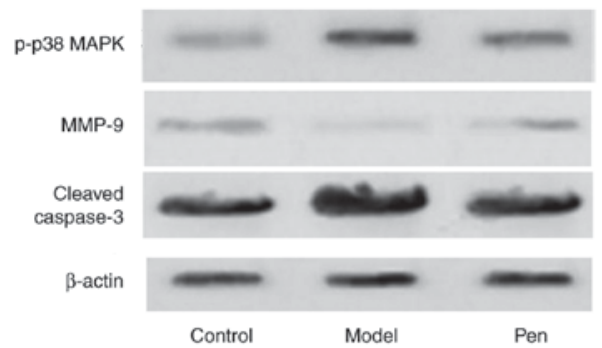

C

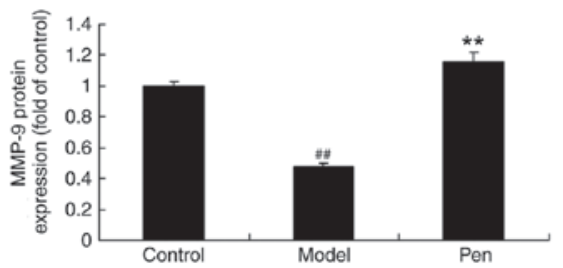

B

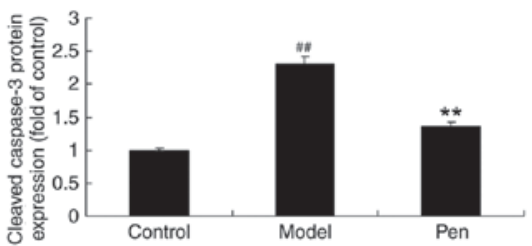

D

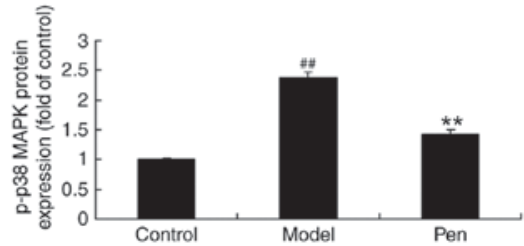

Figure 8. Pentoxifylline treatment affects cleaved caspase-3, MMP-9 and p-p38 MAPK protein expression in cerebral IRI rats. (A) Representative western blot demonstrating the effects of pentoxifylline on cleaved caspase-3, MMP-9 and p-p38 MAPK protein expression. Densitometric and statistical analysis of (B) cleaved caspase-3, (C) MMP-9 and (D) p-p38 MAPK protein expression in cerebral IRI rats. ${ }^{\# \#} \mathrm{P}<0.01$ vs. control group; ${ }^{* *} \mathrm{P}<0.01$ vs. model group. MMP, matrix metallopeptidase; p-, phosphorylated-; MAPK, mitogen-activated protein kinase; IRI, ischemia reperfusion injury; control, sham-operated group; model, cerebral ischemia reperfusion injury model group; pen, pentoxifylline-treated group.

injury and closely associated with the prognosis of cerebral ischemia $(21,23)$. Oxidative damage and the mechanism of death in ischemic neurons has received increased attention in research. The results of the current study demonstrated that pretreatment with pentoxifylline significantly suppressed IL-6 and TNF- $\alpha$ levels, inhibited MDA and increased SOD levels, and reduced COX-2 mRNA and protein expression in cerebral IRI rats. Marques et al (24) reported that pentoxifylline may 
attenuate the inflammatory process and apoptosis via cleaved caspase-3 and COX-2 in rats with intestinal IRI. In addition, Mayyas et al (25) observed that pentoxifylline suppressed myocardial oxidative status following intake of a western diet.

MMP-9 is a type of zinc-dependent proteolytic enzyme (26). In the neuroinflammation reaction, it is released by the stimulation of cytokines and immediate-early factors, reacts to various proinflammatory stimuli and is involved in the inflammatory reaction and pathophysiological process of various nervous system diseases (27). MMP-9 has an important role in the physiological and pathological processes of the central nervous system (28). A previous study indicated that MMP-9 also has an important role in the development of ischemic cerebrovascular disease (29). It has been demonstrated that MMP-9 is expressed in the hippocampus, cortex and striatum of normal brain tissue, although the expression level is very low (30). Following cerebral IRI injury, the expression of MMP-9 in the ischemic brain tissue is reported to be markedly enhanced (30). Notably, the present study demonstrated that treatment with pentoxifylline significantly reduced the iNOS mRNA and protein expression, and induced MMP-9 protein expression in cerebral IRI rats. Garcia et al (31) reported that pentoxifylline decreased glycemia levels through suppression of NOS and COX-2 expression in the pancreas of diabetic rats. In addition, de Campos et al (32) demonstrated that pentoxifylline attenuated pulmonary inflammation via MMP-9 in experimental acute pancreatitis.

A previous study confirmed that the p38 MAPK signaling pathway is closely associated with nerve cell damage (33). During cerebral IRI, p38 MAPK is activated, and the inhibition of p38 MAPK may protect nerve cells from IRI (34). Activation of the p38 MAPK pathway is reported to regulate nitric oxide levels by influencing the expression of iNOS (35). Nitric oxide is an important factor that affects the expression of MMP-9, which directly degrades collagen IV and subsequently leads to increases in blood brain barrier permeability and the exosmosis of IgG from the blood vessel (35). The results of the current study indicate that treatment with pentoxifylline significantly attenuated caspase-3 and p-p38 protein expression in cerebral IRI rats. Costantini et al (36) reported that pentoxifylline suppressed leukoreduced stored blood-induced neutrophil activation via p38 MAPK and extracellular signal-regulated kinase phosphorylation.

In conclusion, the results of the present study demonstrated that pentoxifylline inhibits the neurological deficit score and cerebral infarct volume in cerebral IRI rats, which may occur via anti-inflammation, antioxidation and antiapoptotic mechanisms as pentoxifylline suppressed COX-2 expression, increased MMP-9 expression and downregulated p38 pathways in cerebral IRI rats in vivo. Further research is required to evaluate the potential beneficial effect of pentoxifylline on cerebral IRI in the clinic.

\section{References}

1. Martins VL, Caley MP, Moore K, Szentpetery Z, Marsh ST, Murrell DF, Kim MH, Avari M, McGrath JA, Cerio R, et al: Suppression of TGF $\beta$ and angiogenesis by type VII collagen in cutaneous SCC. J Natl Cancer Inst 108: pii: djv293, 2015.

2. Zhao Y, Wang D, Xu T, Liu P, Cao Y, Wang Y, Yang X, Xu X, Wang $\mathrm{X}$ and Niu $\mathrm{H}$ : Bladder cancer cells re-educate TAMs through lactate shuttling in the microfluidic cancer microenvironment. Oncotarget 6: 39196-39210, 2015.
3. Rodriguez DA, de Lima RF, Campos MS, Costa JR, Biancardi MF, Marques MR, Taboga SR and Santos FCA: Intrauterine exposure to bisphenol A promotes different effects in both neonatal and adult prostate of male and female gerbils (Meriones unguiculatus). Environ Toxicol 31: 1740-1750, 2016.

4. Khoo NK, Cantu-Medellin N, St Croix C and Kelley EE: In vivo immuno-spin trapping: Imaging the footprints of oxidative stress. Curr Protoc Cytom 74: 12.42.1-11, 2015.

5. Yue K, Trujillo-de Santiago G, Alvarez MM, Tamayol A Annabi N and Khademhosseini A: Synthesis, properties, and biomedical applications of gelatin methacryloyl (GelMA) hydrogels. Biomaterials 73: 254-271, 2015.

6. Yan Y, Martin LM, Bosco DB, Bundy JL, Nowakowski RS, Sang QX and Li Y: Differential effects of acellular embryonic matrices on pluripotent stem cell expansion and neural differentiation. Biomaterials 73: 231-242, 2015.

7. Koyano-Nakagawa N, Shi X, Rasmussen TL, Das S, Walter CA and Garry DJ: Feedback mechanisms regulate Ets variant 2 (Etv2) gene expression and hematoendothelial lineages. J Biol Chem 290: 28107-28119, 2015.

8. Warsinske HC, Ashley SL, Linderman JJ, Moore BB and Kirschner DE: Identifying mechanisms of homeostatic signaling in fibroblast differentiation. Bull Math Biol 77: 1556-1582, 2015.

9. Miyamoto DT,Zheng Y, Wittner BS, Lee RJ,Zhu H, Broderick KT, Desai R, Fox DB, Brannigan BW, Trautwein J, et al: RNA-Seq of single prostate CTCs implicates noncanonical Wnt signaling in antiandrogen resistance. Science 349: 1351-1356, 2015.

10. Gill SE, Rohan M and Mehta S: Role of pulmonary microvascular endothelial cell apoptosis in murine sepsis-induced lung injury in vivo. Respir Res 16: 109, 2015.

11. Yang YR, Kim DH, Seo YK, Park D, Jang HJ, Choi SY, Lee YH, Lee GH, Nakajima K, Taniguchi N, et al: Elevated $\mathrm{O}-$ GlcNAcylation promotes colonic inflammation and tumorigenesis by modulating NF- $\mathrm{KB}$ signaling. Oncotarget 6: 12529-12542, 2015.

12. Thurber AE, Omenetto FG and Kaplan DL: In vivo bioresponses to silk proteins. Biomaterials 71: 145-157, 2015.

13. Ji H, Tang H, Lin H, Mao J, Gao L, Liu J and Wu T: Rho/Rock cross-talks with transforming growth factor- $\beta /$ Smad pathway participates in lung fibroblast-myofibroblast differentiation. Biomed Rep 2: 787-792, 2014.

14. Deng H, Zuo X, Zhang J, Liu X, Liu L, Xu Q, Wu Z and Ji A: A-lipoic acid protects against cerebral ischemia/reperfusion-induced injury in rats. Mol Med Rep 11: 3659-3665, 2015.

15. Livak KJ and Schmittgen TD: Analysis of relative gene expression data using real-time quantitative PCR and the 2(-Delta Delta C(T)) method. Methods 25: 402-408, 2001.

16. Saiardi A, Guillermier C, Loss O, Poczatek JC and Lechene C: Quantitative imaging of inositol distribution in yeast using multi-isotope imaging mass spectrometry (MIMS). Surf Interface Anal 46 (Suppl 1): S169-S172, 2014.

17. Hosseini Y, Agah M and Verbridge SS: Endothelial cell sensing, restructuring, and invasion in collagen hydrogel structures. Integr Biol (Camb) 7: 1432-1441, 2015.

18. Siddiqui A, Bhaumik D, Chinta SJ, Rane A, Rajagopalan S, Lieu CA, Lithgow GJ and Andersen JK: Mitochondrial quality control via the PGC1 $\alpha$-TFEB signaling pathway is compromised by parkin Q311X mutation but independently restored by rapamycin. J Neurosci 35: 12833-12844, 2015.

19. Bhattacharya P, Pandey AK, Paul S, Patnaik R and Yavagal DR: Aquaporin-4 inhibition mediates piroxicam-induced neuroprotection against focal cerebral ischemia/reperfusion injury in rodents. PLoS One 8: e73481, 2013.

20. Bassuk JA, Wu D, Lozano H, Arias J, Kurlansky P, Lamas GA and Adams JA: Non-selective cyclooxygenase inhibition before periodic acceleration ( $\mathrm{pGz}$ ) cardiopulmonary resuscitation (CPR) in a porcine model of ventricular fibrillation. Resuscitation 77: 250-257, 2008.

21. Wang T,Zhai L, Zhang H, Zhao L and Guo Y: Picroside II Inhibits the MEK-ERK1/2-COX2 signal pathway to prevent cerebral ischemic injury in rats. J Mol Neurosci 57: 335-351, 2015.

22. Brzozowski T, Konturek PC, Konturek SJ, Pajdo R, Kwiecien S, Pawlik M, Drozdowicz D, Sliwowski Z and Pawlik WW: Ischemic preconditioning of remote organs attenuates gastric ischemia-reperfusion injury through involvement of prostaglandins and sensory nerves. Eur J Pharmacol 499: 201-213, 2004.

23. Salinas G, Rangasetty UC, Uretsky BF and Birnbaum Y: The cycloxygenase 2 (COX-2) story: It's time to explain, not inflame. J Cardiovasc Pharmacol Ther 12: 98-111, 2007. 
24. Marques GM, Rasslan R, Belon AR, Carvalho JG, Felice Neto R, Rasslan S, Utiyama EM and Montero EF: Pentoxifylline associated to hypertonic saline solution attenuates inflammatory process and apoptosis after intestinal ischemia/reperfusion in rats. Acta Cir Bras 29: 735-741, 2014.

25. Mayyas F, Alzoubi KH and Al-Taleb Z: An evaluation of the effect of pentoxifylline on blood pressure and myocardial oxidative status following intake of western diet. Clin Exp Hypertens 37: 666-673, 2015.

26. Xin L, Hou Q, Xiong QI and Ding X: Association between matrix metalloproteinase-2 and matrix metalloproteinase- 9 polymorphisms and endometriosis: A systematic review and meta-analysis. Biomed Rep 3: 559-565, 2015.

27. Sun J, Zhu Y, Zhang L and Ma Y: Effects of xuelian injection on cerebral TNF- $\alpha$, IL-1 $\beta$ and MMP-9 in rats experienced focal cerebral ischemia/reperfusion. Int J Clin Exp Med 7: 2632-2638, 2014.

28. Zheng M, Wei J, Tang Y, Yang C, Wei Y, Yin X and Liu Q: ApoE-deficient promotes blood-brain barrier disruption in experimental autoimmune encephalomyelitis via alteration of MMP-9. J Mol Neurosci 54: 282-290, 2014.

29. Kim SJ and Lee SR: Protective effect of melatonin against transient global cerebral ischemia-induced neuronal cell damage via inhibition of matrix metalloproteinase-9. Life Sci 94: 8-16, 2014.

30. Xin H, Liang W, Mang J, Lin L, Guo N, Zhang F and Xu Z: Relationship of gelatinases-tight junction proteins and blood-brain barrier permeability in the early stage of cerebral ischemia and reperfusion. Neural Regen Res 7: 2405-2412, 2012.
31. Garcia FA, Pinto SF, Cavalcante AF, Lucetti LT, Menezes SM, Felipe CF, Alves AP, Brito GA, Cerqueira GS and Viana GS: Pentoxifylline decreases glycemia levels and TNF-alpha, iNOS and COX-2 expressions in diabetic rat pancreas. Springerplus 3: $283,2014$.

32. de Campos T, Deree J, Martins JO, Loomis WH, Shenvi E, Putnam JG and Coimbra R: Pentoxifylline attenuates pulmonary inflammation and neutrophil activation in experimental acute pancreatitis. Pancreas 37: 42-49, 2008.

33. Liu XW, Ji EF, He P, Xing RX, Tian BX and Li XD: Protective effects of the p38 MAPK inhibitor SB203580 on NMDA-induced injury in primary cerebral cortical neurons. Mol Med Rep 10: 1942-1948, 2014

34. Wu MH, Chio CC, Tsai KJ, Chang CP, Lin NK, Huang CC and Lin MT: Obesity exacerbates rat cerebral ischemic injury through enhancing ischemic adiponectin-containing neuronal apoptosis. Mol Neurobiol 53: 3702-3713, 2016.

35. Madhyastha R, Madhyastha $H$, Nakajima $Y$, Omura $S$ and Maruyama M: MicroRNA signature in diabetic wound healing: Promotive role of miR-21 in fibroblast migration. Int Wound J 9: 355-361, 2012.

36. Costantini TW, Deree J, Martins JO, Loomis WH, Bansal V and Coimbra R: Pentoxifylline attenuates leukoreduced stored blood-induced neutrophil activation through inhibition of mitogen-activated protein kinases. Immunopharmacol Immunotoxicol 32: 74-81, 2010. 